\title{
SCIL-VP: a multi-purpose visual programming environment*
}

\author{
Dennis Koelma, Richard van Balen, Arnold Smeulders
}

\author{
University of Amsterdam
}

\begin{abstract}
This paper presents a multi purpose visual programming environment called SCIL-VP. In SCIL-VP programs are specified as data flow graphs consisting of arbitrary functions operating on arbitrary data objects. The environment enables a mixture of visual and textual programming. The visual programming interface is generated at runtime from information in a command description file. As a consequence the library of functions is easy to extend, even by laymen. The data objects are also extensible but this requires elementary programming skills. The setup of extensibility makes the environment independent of an application domain. It also opens the way to the combination of different application domains.
\end{abstract}

\section{Introduction}

The use of computers through specialized software packages is increasing rapidly to such a degree that a typical end-user is no longer able to make the step to programming a computer. Programming a computer in a conventional programming language, i.e. a textual one, requires a precise sense for logic which may not suit every end-user. Visual programming may help to bridge the gap by making programming easier. Experienced programmers may also benefit from the advantages visual programming has over conventional textual programming. It enables them to concentrate more on solving the problem and less on writing the program.

*This research was supported by STW project AWI99-1691

Permission to copy without foe all or part of this material is granted provided that the copies are not made or distributed for direct commercial advantage, the ACM copyright notice and the title of the publication and its date appear, and notice is given that copying is by permission of the Association for Computing Machinery. To copy otherwise, or to republish, requires a foe and/or specific permission.

- 1992 ACM 0-89791-502-X/92/0002/1188...\$1.50
Visual programming languages can be classified according to different taxonomies [1-3]. We will stay away from such a discussion but use the operational definition that the essence of visual programming is the use of visual expressions (such as drawings or icons) in the process of programming.

Essentially different from visual programming is program visualization where the program is specified in a conventional, textual manner and the visual representation is used to illustrate some aspect of the program e.g. the algorithm, the data structures or the dynamic behavior of the program [4-9]. These approaches are not considered here as they require the program to be specified in an textual language and thus would not help the end-user in mastering the machine.

Visual programming languages have many advantages over textual programming languages $[1,2,10-12]$. Their two-dimensional layout and use of icons seem to be closer to the human way of thinking [13]. This then simplifies the translation of the representation used in the mind while thinking about a problem to the representation used in programming a problem. The shorter translation distance makes visual languages easier to comprehend, learn, use, and remember. The use of pictorial elements is important because frequently pictures convey more meaning than text: a single picture is often worth more than a thousand words (the converse may also be true). Furthermore, the combination of a twodimensional layout and the use of animation visualizes the execution of a program to help in debugging and program optimalization. Finally, the two-dimensional layout facilitates the detection of potential concurrency in a program for parallel computation.

A disadvantage of visual programming is the large space it requires on the screen to create a program. This can be solved only partially through the use of scrolling and/or various abstraction mechanisms such as a hierarchical structure in the program. This problem is most 
obvious in the general purpose visual programming languages [14-22], the visual languages used as an interface to a textual programming language $[23-25]$ and the concurrent visual programming languages [26-28]. They all use low level basic elements, functions and data objects, to develop programs. Although these languages can be used in different application domains, the low level basic elements often make it be too cumbersome to write a non-trivial program, especially for laymen. Combining the visual language with a textual language [29-32] reduces the space problem and allows for the use of textual programming in those part of a problem that are hard or impossible to solve using the visual language. However, textual languages require more programming skills and do not have the advantages of the visual languages so they can not assist the laymen. They are only of interest to an expert because experts like to have an escape possibility from the particular language they are using.

Other visual programming environments use high level basic elements to allow for the development of small, yet powerful, programs. These environments are used in database processing [33-37], in image processing [3840], in educational software [41-44], as a command shell $[45,46]$, in user interface design [47], in expert systems [48], in security systems [49], in matrix computation [50], etc. The disadvantage of these environments is that they can only be used in the small application domain they were developed for. The visual programming interface is hard coded and thus the environment is not easily modified and not extensible to other application domains.

It is felt that visual programming is not generally accepted because the approaches mentioned above all have serious drawbacks: writing a program in a general purpose visual programming language is too cumbersome and using specialized visual programming environments limits the use to a small application domain. Combining the approaches could be a solution. The visual programming environment presented here adheres to the concept of high level basic elements in the construction of programs since they are essential for end-users. To avoid being trapped in a small application domain the environment was made easily extensible for new basic elements. To give the expert his escape possibility the combination with a textual language is also provided for. The KHOROS system $[51,52]$ uses a similar approach but requires the basic elements to be developed especially for the environment. This makes it more difficult to add existing software libraries to the environment.

Section 2 gives an overview of the SCIL environment upon which the presented visual programming environment was build. The concept of SCIL-VP is described in section 3 . Some implementational aspects are described in section 4. Section 5 shows the development of a sample program. Section 6 concludes with a discussion of the presented environment.

\section{The SCIL environment}

The idea for SCIL-VP originated in the SCIL environment $[53,54]$. SCIL is a multi-layer software development environment based on a $\mathrm{C}$ interpreter. It gives the user easy access to functions of a software library on multiple levels. At the first level, functions from a library can be called from within the interpreter by typing ordinary C-function calls and pressing the return key. Functions can also be executed through a command expander. At this level the user can abbreviate function names, disregard the proper C-syntax, ask for parameters and rely on a mechanism that supplies default values for parameters that were left out. After the user presses the return key, the command is expanded into a legal C-statement and executed. At the third level functions can be picked from a menu, resulting in a dialogue box. The user can alter the parameters of the function in this dialogue box and execute the function by pressing the Do-It button. All levels can be used simultaneously. The user interface is built at start-up from a command description file consisting of a menu tree and a description of every function with its arguments. All levels use the command description file to access the functions of a library, thus by adding a description of a function to this file the function can be used at each level.

The various levels of the SCIL environment offer an increasing level of support for inexperienced users but at the highest level the programming possibilities of the $\mathrm{C}$ interpreter level disappear. Combining ease of use and programmability in some way would allow inexperienced users to create non-trivial programs quickly by using the high level functions from a library. This observation led to the development of the presented visual programming environment. This new layer has both a high level of support for an inexperienced user and has programming capabilities.

\section{Design of SCIL-VP}

The intent of SCIL-VP is to create small, yet powerful programs by combining high level functions from a user specified library from one or more application domains. This intent implies that the visual language must be able to express an operation on data clearly. The language is not to be an algorithmic language because these 
kinds of languages require the inexperienced user to focus too much on the process of programming. This can lead to what is termed "analysis paralysis": the means become the ends as you forget what you wanted to get out of the computer and become wrapped up in the process of getting it out [55]. These observations lead to the choice of a data flow language.

The data flow language used in SCIL-VP does not support the use of control structures. Although control structures have been added to data flow languages [14, $15,17,29]$, it is hard, if not impossible, to make them flexible enough and still easy to use. The data flow language is combined with the textual language $C$ to give a more experienced programmer the possibility to address problems that cannot be solved using the controlless data flow language only. It also gives him the possibility to escape from the visual language in those parts of a problem that can be expressed more clearly in a textual language.

The functionality of the system is provided by the functions from the user specified library (or libraries). The functions are the building blocks from which the user starts to construct a visual program. To keep the environment application independent, the addition of a new library of functions should be simple, where only a compact description of each function has to be supplied. This description includes the name of the function and a list of parameters, if present. The only restriction imposed upon the functions by SCIL-VP is that they must be C-callable functions. For most systems this does not necessarily imply that the functions are written in C. There should be no restrictions to possibilities of a function for user interaction: it should have normal access to the standard input/output of a terminal and to the window system.

The use of arbitrary functions requires the support of arbitrary data objects. Therefor, the environment is designed such that it is extensible in its data objects. To accomplish this the environment makes use of only a limited number of functions in the communication with the data objects. A data object should be able to represent anything as long as it can be accessed through (a reference to) an object. There should be no restriction to the complexity of an object.

A visual and hierarchical overview of the functions is to be provided by the library handler. This overview should reflect the ordering of the functions in groups and subgroups. As always the (sub) groups and the functions are represented by icons.

The user should be able to use multiple worksheets in the development of a visual program. These worksheets should also be able to reflect the hierarchical structure of a program. Upon completion of the development of a visual program it should be possible to execute the program without the visual programming environment.

\section{Implementation}

SCIL-VP was written in C and developed on Sun workstations using the $\mathrm{X}$-window system.

\subsection{Functions}

The application independency of the SCIL-VP environment is largely due to the command description file. In this file the user can specify a menu tree with functions. The menu tree can be specified by naming a menu together with its parent. For example, the line "\$Manipulation \$Image" means that the menu Manipulation is a submenu of the Image menu (the dollar sign stands for menu). The functions from the user-specified library (or libraries), which make up the functionality of the SCIL-VP system, are also described in this file. The description of each function consists of the following items:

func The name of the function.

(sub)menu(s) The (sub)group(s) the function is associated with.

bitmap The icon of the function. This is the name of the file with a bitmap of the icon of arbitrary dimensions. The file is created with a standard $X$ bitmap editors. If no file is specified an icon will be created showing the name of the function as a text string.

ret_val The type of the data object that is the return value of the function. This item does not have to be specified if the return value is to be ignored.

args The list of parameters of the function. The description of each parameter consists of the following items:

data_type The data object type of the parameter, e.g. integer, string.

par_type The type of the parameter. Four types of parameters exist: input, output, input/output and control parameters. The input parameters are shown as a pin on the left side of the function (see figure 1). The output parameters are shown on the right side. The input/output parameters have pins on both 


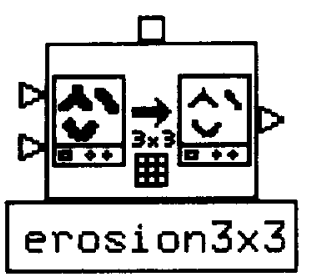

Figure 1: An example of an icon of the function erosion $3 x 3$

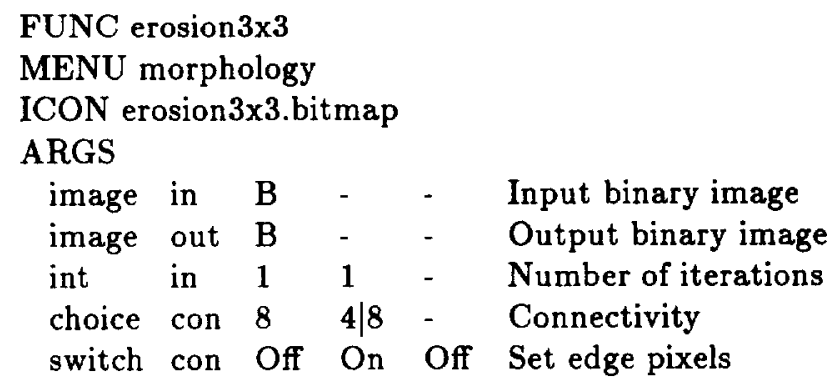

Figure 2: The description of the function erosion $3 x 3$

sides of the function. Control parameters are parameters that are not altered very often. They are not represented by pins to avoid cluttering of the screen but they can be altered through a dialogue box at any moment. The presence of control parameters is indicated by a little square at the top of the function.

default The default value of the parameter.

min The minimum admitted value of the parameter or a set of legal values.

max The maximum admitted value of the parameter.

prompt Short description of the parameter.

Figure 2 gives an example of the description of a function.

The command description file is a plain ASCII file that can be altered by any text editor. Altering the command description file does not enforce recompilation of the program because the file is read during the SCIL-VP environment startup.

\subsection{Data objects}

The data objects represent the entities on which the library of functions is operating. In an image processing library, for example, these entities are images. A data object is (a reference to) a data structure. This structure must contain all data relevant to an entity but can be of arbitrary complexity. An image object, for example, does not only contain the image data but also other information like the name of the image, its sizes, its type, a reference to the window it is displayed in, etc.

Four types of data objects are defined in the current version of SCIL-VP: integers, doubles, strings and images. The environment makes use of only three C-functions in its communication with the data objects. These functions are to create, copy and destroy a data object. A new type of data object can be defined by providing these 3 functions for the specific type as a C-program. By taking the $\mathrm{C}$-code of the functions of an existing type of data objects as an example, it is considered not to be too difficult to add new types.

Beside these three functions two more functions can be added to generate or monitor a data object, but these functions are not compulsory. These functions can be used to start and end a data flow graph.

\subsection{Library handler}

The library handler (see figure 3 ) gives a hierarchical overview of the functions known to the environment. Groups are distinguished from functions by their thick border. At the top of the window the path to the current group (Image in figure 3 ) is shown. In the remainder of the window the subgroups and functions of the current group are shown. The user can move to another group by selecting its icon in the path to the current group or by selecting its icon in the current group. A function can be selected from the library by clicking its icon (convert in figure 3 ).

\subsection{Worksheets}

A worksheet (see figure 4) consists of a control panel and a workspace. Only part of the workspace is visible as indicated by the scroll bars. The visible part of the workspace can have any size as long as it fits on the screen. The size of the workspace itself is unlimited.

\subsubsection{The worksheet editor}

In the editor the user can select objects (functions, connections, pins and comments) by pointing at them and pressing the left mouse button. When an input pin and output pin are selected they are connected by a straight, thick line. The straight line is used to avoid screen clut- 


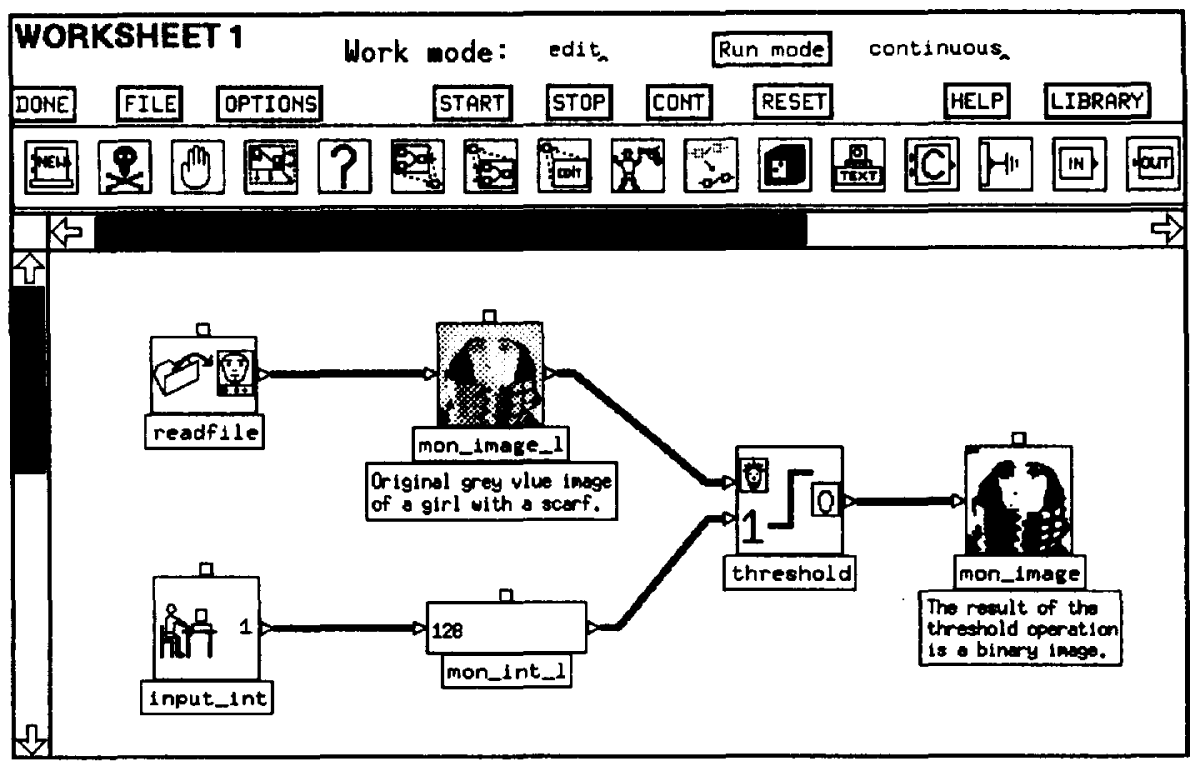

Figure 4: Example of a worksheet

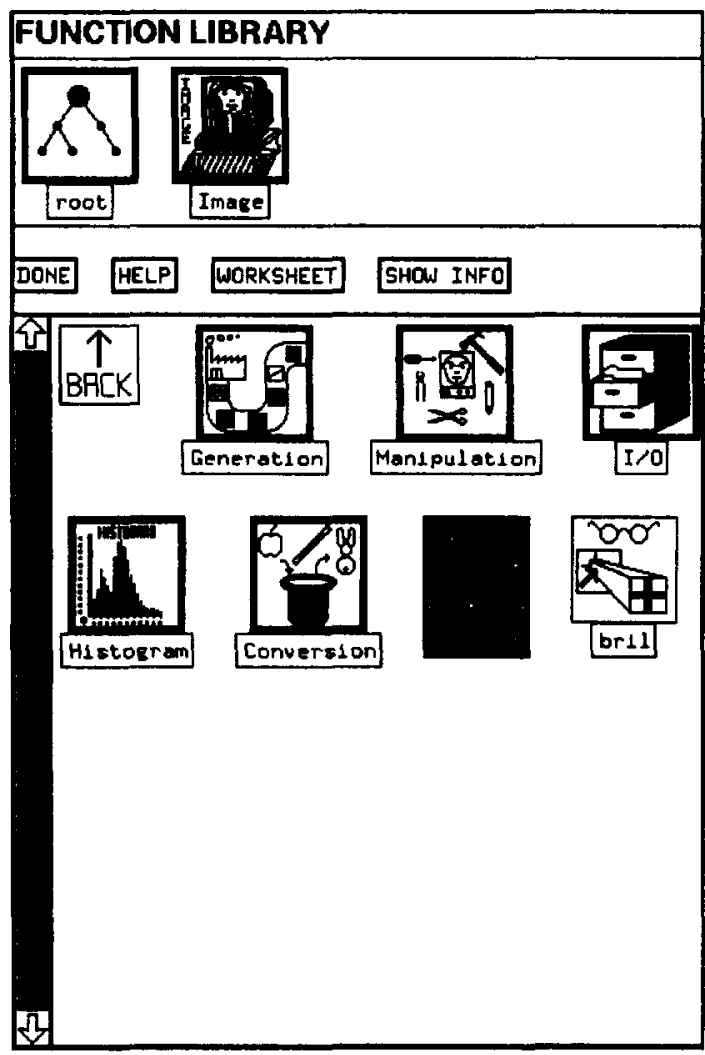

Figure 3: An example of the face of the library handler tering. An attempt to make a connection results in an error when the selected pins have incompatible data types. An output pin can be connected to many different input pins, but only one connection is allowed on an input pin.

The editor also has a series of special edit commands. These commands are represented by the icons above the workspace. They can be executed by first selecting the icon of the command and then the objects the command has to be executed on or vice versa. Both methods exist only when the nature of the command allows for it. Examples of the edit commands are:

new In this mode a copy of the selected function in the library is placed on the worksheet when the left mouse button is pressed.

info The user can ask for help or information on an object. When a function is selected the standard SCIL help window pops up, showing information about the function.

compose Several functions can be grouped together to form a new one. The user is allowed to create a bitmap of arbitrary dimensions for the new function. In this way a hierarchical structure is created. User defined functions behave in exactly the same way as all other functions do.

decompose A user defined function can be split again into its original components. 
udf-edit A user defined function can be edited in a separate window. The editor behaves like a normal worksheet with the same editing capabilities. It also allows the user to add or remove parameters of the function.

data safe A data safe can be placed on a connection to keep the data valid. The use of this function is explained in more detail in the next section.

comment The user can add comments to a function. These comments are displayed below the icon of the function. These comments (and the function names below the icons) can be made invisible by a system option.

C-icon Add a C-icon to the worksheet as described in section 4.4.3.

\subsubsection{Execution}

Once the program has been composed it can be executed by pressing the start button. The starting point of the execution can be influenced by selecting a function before pressing this button. Since the program is interpreted the execution starts immediately. The order in which the functions are executed is a combination of data and demand driven. If there is a function that has valid data on all input pins then it is executed (data driven). If a function does not have valid data on all input pins it asks its predecessor for data (this is the demand driven portion). The predecessor will try to create the data or pass the demand to their predecessor. After the function has been executed all data at its input pins is destroyed, unless some other function still needs it.

The function under execution is highlighted so the user can follow the execution. Optionally the executed function is positioned at the center of the visible part of the workspace. The execution can be done in a continuous mode or in step mode. This mode can be changed during execution. The execution stops if there are no more functions to be executed, if there is a loop in the program, or if the user presses the stop button. Once the program has halted the worksheet returns to the edit mode. The execution of the program can be continued later if the program has not been altered in the mean time.

\subsubsection{Combining with C-code}

The visual language can be combined with the textual language $\mathrm{C}$ by means of the $\mathrm{C}$-icon. This icon can be used as a normal function in the data flow program.
Once the $\mathrm{C}$-icon is reached, the associated $\mathrm{C}$-code associated is executed. The $\mathrm{C}$-icon is manipulated by clicking the right mouse button on the function. This will pop up a menu that allows the user to add pins, destroy pins, and change the $\mathrm{C}$-code of the function.

The C-code may contain variable declarations and $\mathrm{C}$ statements. The data of the input and output pins is available through predefined variables, e.g. INPUT1 contains the data of the first input pin.

Once a visual program has been developed and working correctly it can be saved as an ordinary C-program. This allows the user to execute the program without the SCIL-VP environment.

\section{Sample session}

This section describes the development of a sample program. The program shows how an image with nonuniform illumination can be segmented with global thresholding. The meaning of the program may not be entirely clear but that does not matter at this point. First the image must be loaded so the readfile function is selected from the library and placed on the worksheet. The readfile function has a control parameter that specifies the file to be read. Selecting the square button above the icon generates a dialogue box that can be used to alter the control parameter (figure 5). To see the image a monitor can be connected to the readfile function. The connection is made by selecting both pins (figure 6 ).

Figure 7 shows that global thresholding is not possible on the original image. First the shading must be removed from the image. This is done here by subtracting the result of a function called grey value opening (an erosion followed by a dilation) from the original image. After the shading correction the image is segmented using a global thresholding function (figure 8). The components for the shading correction can be combined into a single function to create a hierarchical structure in the program (figure 9 ). The user specifies a name for the new function and creates a bitmap to represent its icon. The function can be edited in a separate window (figure 10).

When a part of the program is working correctly it does not have to be executed over and over again during the development of the rest of the program. This can be avoided by placing a data safe on the connection between the working part and the rest of the program. This data safe will keep the data on the connection valid. For example, placing a data safe on the connection between shading_correction and isodata_threshold, and starting the execution at isodata_threshold will pre- 
vent shading_correction from being executed over and over again.

\section{Discussion}

SCIL-VP allows users to write non-trivial programs by combining arbitrary, high level functions into a data flow graph. The use of data flow graphs in programming enhances the understanding of the operation of a program through its two-dimensional layout and the use of icons. The high level functions in combination with visual programming help to keep the mind of the (inexperienced) user on solving the problem instead of concentrating on the act of programming. The programs have the same expressibility as controlless data flow graphs. In addition, the integration of C-code within an icon in the data flow graph gives a more experienced user the possibility to use the best of both worlds.

A notorious drawback of visual programming is the large space needed on the screen. This cannot be solved completely with a hierarchical structure in the program as the overall structure of the program remains unclear. The application of the image processing library in this paper has shown that it may be hard to create a pictorial abstraction of a function. It has also shown the importance of good icons. Good icons can help in understanding and remembering but bad icons lead to confusion.

The SCL-VP environment distinguishes itself from other visual programming environments in the possibilities to extend the environment with new functions. SCIL-VP is easily extended due to the use of the command description file and due to the restricted number of data communication functions. This enables the use of SCIL-VP in different application domains. It also opens the way to combining the functionality of various application domains.

\section{REFERENCES}

[1] N. C. Shu. Visual programming. Van Nostrand Reinhold Company Inc., 1988.

[2] S.-K. Chang. Visual languages, a tutorial and survey. IEEE Software, pages 29-39, January 1987.

[3] B. A. Myers. Taxonomies of visual programming and program visualization. Journal of Visual Languages and Computing, pages 97-123, March 1990.

[4] B. A. Myers, R. Chandhok, and A. Sareen. Automatic data visualization for novice pascal programmers. In Proceedings of the 1988 Workshop on Visual Languages, pages 192-198, 1988.
[5] V. Haarslev and R. Moller. Visualization of experimental systems. In Proceedings of the 1988 Workshop on Visual Languages, pages 175-182, 1988.

[6] H. Lieberman. A three-dimensional representation for program execution. In Proceedings of the 1989 Workshop on Visual Languages, pages 111$116,1989$.

[7] S. P. Reiss, S. Meyers, and C. Duby. Using GELO to visualize software systems. In Proceedings of the Second Annual Symposium on User Interface Software and Technology, pages 149-157, 1989.

[8] M. H. Brown and R. Sedgewick. A system for algorithm animation. ACM Computer Graphics (Proceedings SIGGRAPH '84), pages 177-186, July 1984 .

[9] M. H. Brown. Exploring algorithms using Balsa-II. IEEE Computer, pages 14-36, May 1988.

[10] E. P. Glinert, M. E. Kopache, and D. W. McIntyre. Exploring the general-purpose visual alternative. Journal of Visual Languages and Computing, pages 3-39, March 1990.

[11] G. Raeder. A survey of current graphical programming techniques. IEEE Computer, pages 11-25, August 1985.

[12] S. L. Tanimoto and E. P. Glinert. Designing iconic programming systems: representation and learnability. In Proceedings of the 1986 Workshop on Visual Languages, pages 54-60, 1986.

[13] D. C. Smith. Pygmalion: a creative programming environment. Report no. stan-cs-75-499, Stanford, June 1975.

[14] P. T. Cox, F. R. Giles, and T. Pietrzykowski. Prograph: a step towards liberating programming from textual conditioning. In Proceedings of the 1989 Workshop on Visual Languages, pages 150-156, 1989.

[15] D. N. Smith. Visual programming in the interface construction set. In Proceedings of the 1988 Workshop on Visual Languages, pages 109-120, 1988.

[16] D. Ingalls, S. Wallace, Y.-Y. Chow, F. Ludolph, and K. Doyle. Fabrik: a visual programming environment. In OOPSLA '88 Conference Proceedings, pages 176-190, 1988.

[17] T. Ichikawa and M. Hirakawa. Visual programming - toward realization of user-friendly programming environments. In Proceedings 2nd Fall Joint Computer Conference, pages 129-137, 1987. 


\section{readfile}

Filenane: trui.1m

DO IT CANCEL

Figure 5: An example of a dialogue box to set a control parameter

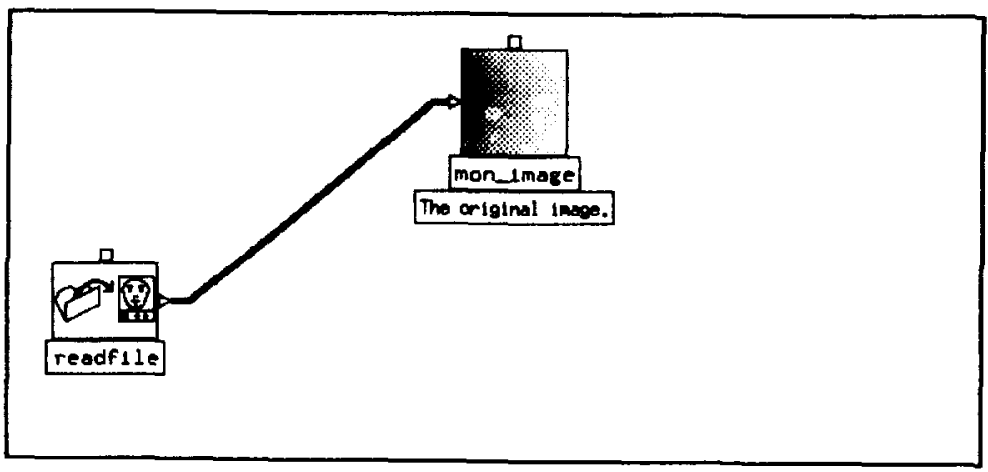

Figure 6: A connection between two functions

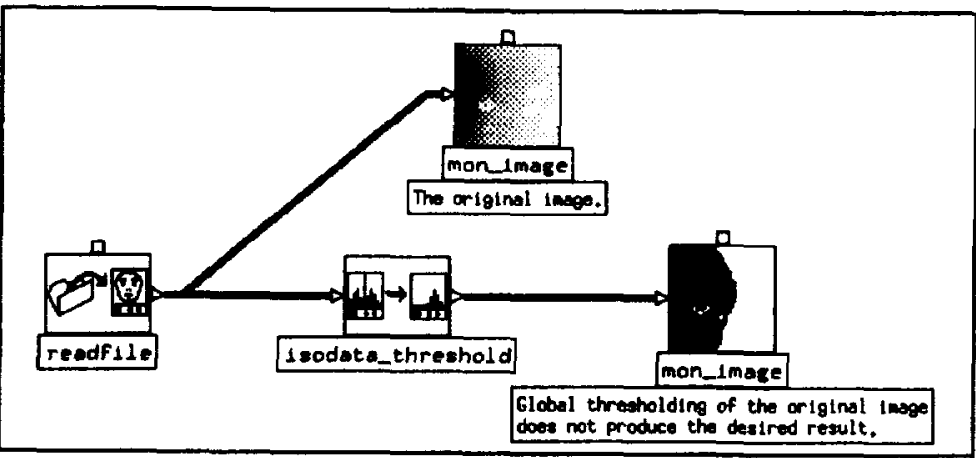

Figure 7: Global thresholding of the original image 


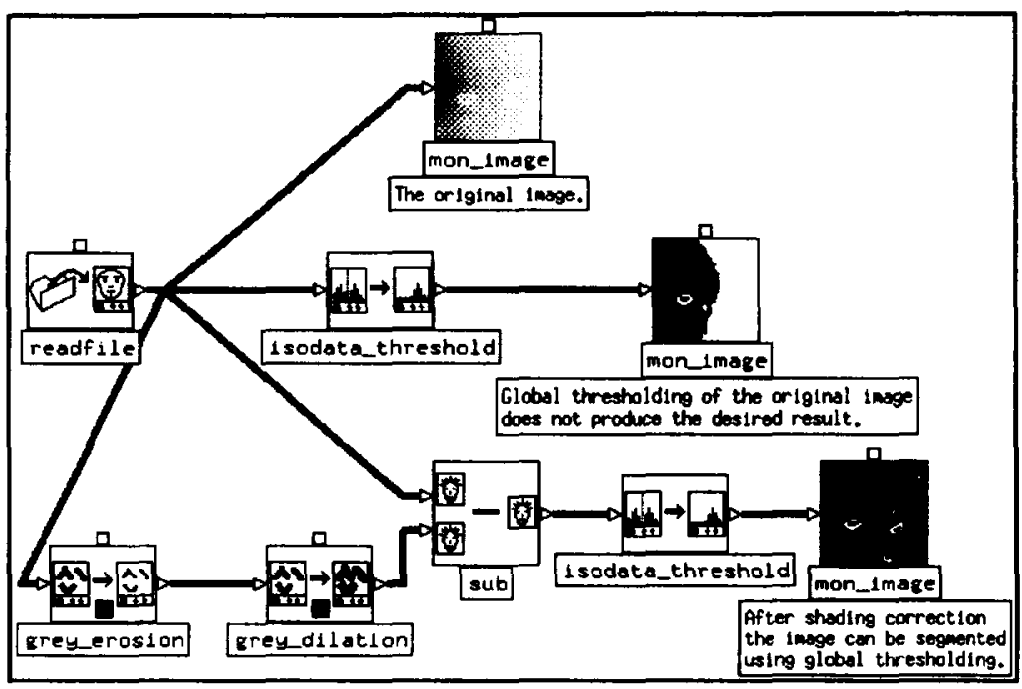

Figure 8: Global thresholding after shading correction

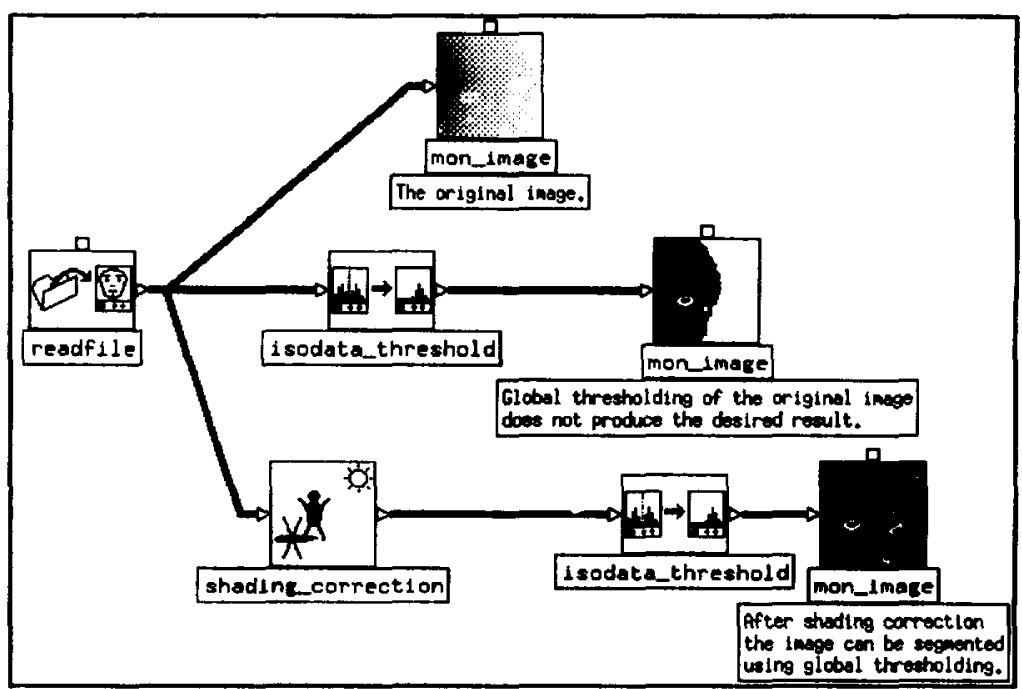

Figure 9: $\mathrm{A}$ user defined function 


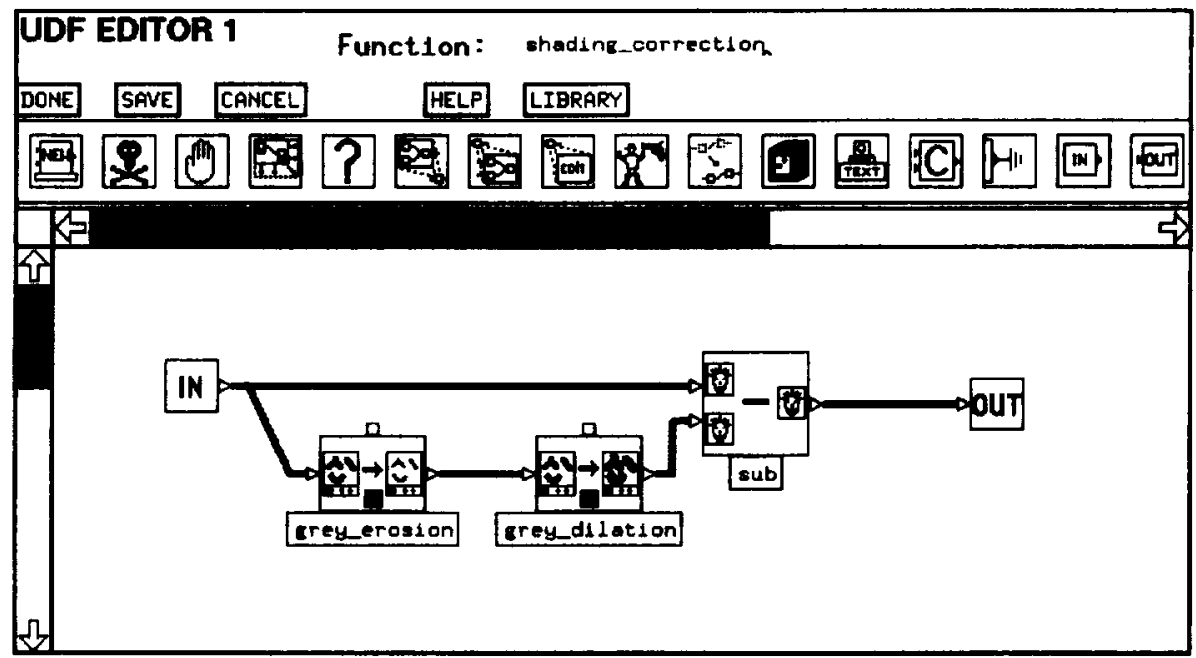

Figure 10: The udf-editor

[18] A. Borning. The programming language aspects of ThingLab, a constraint-oriented simulation laboratory. ACM Transactions on Programming Languages and Systems, pages 353-387, October 1981.

[19] A. Borning. Graphically defining new building blocks in ThingLab. Human-Computer Interaction, 2(4):269-295, 1986.

[20] M. C. Pong and N. Ng. PIGS - a system for programming with interactive graphical support. Software - Practice and Experience, pages 847-855, September 1983.

[21] S. P. Reiss. PECAN: program development systems that support multiple views. IEEE Transactions on Software Engineering, pages 276-285, March 1985.

[22] S. P. Reiss. Working in the Garden environment for conceptual programming. IEEE Software, pages 16-27, November 1987.

[23] M. Edel. The Tinkertoy graphical programming environment. IEEE Transactions on Software Engineering, pages 1110-1116, August 1988.

[24] G. Cattaneo, A. Guercio, S. Levialdi, and G. Tortora. Iconlisp: an example of a visual programming language. In Proceedings of the 1986 Workshop on Visual Languages, pages 22-25, 1986.

[25] J. L. Diaz-Herrera and R. C. Flude. PASCAL/HSD: A graphical programming system. In IEEE Proceedings COMPSAC, pages 723-728, 1980.
[26] M.-C. Pong. A graphical language for concurrent programming. In Proceedings of the 1986 Workshop on Visual Languages, pages 26-33, 1986.

[27] T. Ae, M. Yamashita, and W. C. Cunha. Visual user-interface of a programming system MOPS ${ }^{2}$. In Proceedings of the 1986 Workshop on Visual Languages, pages 44-53, 1986.

[29] P. D. Stotts. The FPG language: visual programming for concurrent computation. In Proceedings of the 1988 International Conference on Parallel Processing, pages 72-79, 1988.

[29] S. Matwin and T. Pietrzykowski. Prograph: a preliminary report. Computer Languages, 10(2):91$126,1985$.

[30] E. P. Glinert. Towards "second generation" interactive, graphical programming environments. In Proceedings of the 1986 Workshop on Visual Languages, pages 61-70, 1986.

[31] M. E. Kopache and E. P. Glinert. $C^{2}$ : a mixed textual/graphical environment for C. In Proceedings of the 1988 Workshop on Visual Languages, pages 231-238, 1988.

[32] K. Kojima, Y. Matsuda, and S. Futatsugi. LIVE - integrating visual and textual programming paradigms. In Proceedings of the 1989 Workshop on Visual Languages, pages 80-85, 1989.

[33] K. Tsuda, M. Hirakawa, M. Tanaka, and T. Ichikawa. IconicBrowser: an iconic retrieval system for object-oriented databases. In Proceedings 
of the 1989 Workshop on Visual Languages, pages 130-137, 1989.

[34] B. Czejdo, D. Embley, and V. Reddy. A visual query language for an ER data model. In Proceedings of the 1989 Workshop on Visual Languages, pages 165-170, 1989.

[35] N. C. Shu. FORMAL: a forms-oriented, visualdirected application development system. IEEE Computer, pages 38-49, August 1985.

[36] G. Rogers. Visual programming with objects and relations. In Proceedings of the 1988 Workshop on Visual Languages, pages 29-36, 1988.

[37] S.-K. Chang. Visual reasoning for information retrieval from very large databases. In Proceedings of the 1989 Workshop on Visual Languages, pages $1-6,1989$.

[38] S.-K. Chang, E. Jungert, S. Levialdi, G. Tortora, and T. Ichikawa. An image processing language with icon assisted navigation. IEEE Transactions on Software Engineering, pages 811-819, August 1985.

[39] S. L. Tanimoto. VIVA: a visual language for image processing. Journal of Visual Languages and Computing, pages 127-139, June 1990.

[40] R. P. Blanford and S. L. Tanimoto. The PyramidCalc system for research in pyramid machine algorithms. In Proceedings of the 1986 Workshop on Visual Languages, pages 138-142, 1986.

[41] E. Corten, H. J. W. Spoelder, F. H. Ullings, F. C. A. Groen, and I. T. Young. SIGNOR: a tool for visualization of concepts of system and signal theory. In Proceedings of the 1989 Workshop on Visual Languages, pages 189-192, 1989.

[42] E. P. Glinert and S. L. Tanimoto. Pict: an interactive graphical programming environment. IEEE Computer, pages 7-25, November 1984.

[43] W. Finzer and L. Gould. Programming by rehearsal. Byte, pages 187-210, June 1984.

[44] J. Roberts, J. Pane, M. Stehlik, and J. Carrasquel. The Design View: a design oriented, high-level visual programming environment. In Proceedings of the 1988 Workshop on Visual Languages, pages 213-220, 1988.

[45] K. Borg. Visual programming and UNIX. In Proceedings of the 1989 Workshop on Visual Languages, pages 74-79, 1989.
[46] T. DeMarco and A. Soceneantu. Syncro: a dataflow command shell for the Lilith/modula computer. In Proceedings of the 7th International Conference on Software Engineering, pages 207-213, 1984.

[47] R. J. K. Jacob. A state transition diagram language for visual porgamming. IEEE Computer, pages 5159, August 1985.

[48] M. A. Musen, L. M. Fagan, and E. H. Shortliffe. Graphical specification of procedural knowledge for an expert system. In Proceedings of the 1986 Workshop on Visual Languages, pages 167-178, 1986.

[49] A. Heydon, M. W. Maimone, J. D. Tygar, J. M. Wing, and A. Moormann Zaremski. Miró tools. In Proceedings of the 1989 Workshop on Visual Languages, pages 86-91, 1989.

[50] R. Yeung. MPL - a graphical programming environment for matrix processing based on logic and constraints. In Proceedings of the 1988 Workshop on Visual Languages, pages 137-143, 1988.

[51] C. S. Williams and J. R. Rasure. A visual language for image processing. In Proceedings of the 1990 Workshop on Visual Languages, pages 86-91, 1990.

[52] J. R. Rasure and C. S. Williams. An integrated data flow visual language and software development environment. Journal of Visual Languages and Computing, pages 217-246, September 1991.

[53] T. K. ten Kate, R. van Balen, A. W. M. Smeulders, F. C. A. Groen, and G. A. den Boer. SCILAIM: a multi-level interactive image processing environment. Pattern Recognition Letters, pages 429-441, June 1990.

[54] University of Ansterdam for the Centre of Image Processing and Pattern Recognition (CBP). SCIL_Image manual, July 1991.

[55] G. D. Brown and D. H. Sefton. The micro vs. the applications logjam. Datamation, pages 96-104, january 1984. 